\title{
Development of Gastrointestinal Mucosal Barrier. II. The Effect of Natural Versus Artificial Feeding on Intestinal Permeability to Macromolecules
}

\author{
J. N. UDALL, "lii P. COLONY, L. FRITZE, K. PANG, J. S. TRIER, AND W. A. WALKER \\ Department of Pediatrics and Medicine, Harvard Medical School, the Children's Service (Pediatric Gastrointestinal \\ and Nutrition Unit). Massachusetts General Hospital, Department of Medicine (Gastroenterology. Brigham and \\ Women's Hospital, Boston, Massachusetts; and Department of Nutrition and Food Sciences, Massachusetts Institute \\ of Technologu. Cambridge, Massachusetts, USA
}

\begin{abstract}
Summary
We have recently reported that the intestinal transport of intact macromolecules into the circulation decreases with age presumably due to maturation of mucosal barrier factors. To extend this observation and determine the effect of natural sersus artificial feeding on maturation of intestinal mucosal "barrier function," we conducted experiments which assessed both macromolecular transport and epithelial cell morphology.

To study barrier function, we gavage fed a physiologic quantity $(100 \mathrm{mg})$ of bovine serum albumin (BSA) to weight-matched breast- and bottle-fed infant rabbits at 1 and 2 wk of age and quantitated intestinal macromolecular transport by measuring circulating plasma concentrations of the intact antigen $4 \mathrm{hr}$ later. A significant decrease $(P<0.02)$ in immunoreactive bovine serum albumin (I-BSA) concentration was noted in breast-fed (6.12 \pm $0.77 \mu \mathrm{g}$ I-BSA per ml plasma) compared with bottle-fed (9.19 \pm $0.93 \mu \mathrm{g}$ I-BSA per ml plasma) animals at one wk. However, at 2 wk, no difference could be demonstrated between the two groups. Furthermore, small intestinal morphology evaluated by light and electron microscopy was similar in both groups at each age.

To determine if the lower plasma I-BSA noted at one wk in naturally fed animals was related to the presence of anti-BSA antibodies in breast milk and/or in plasma of the pups, breast milk and plasma from the breast-fed animals was evaluated by counterimmunoelectrophoresis and hemagglutination. No anti-BSA antibodies were detected. Moreover, plasma from breast- and artificially fed rabbits not gavage fed BSA contained no I-BSA.

These data suggest that intestinal transport of antigens in the immediate neonatal period is decreased earlier in breast- as compared to bottle-fed animals. Therefore, we suggest that breast milk may exert a protective function to control the transport of potentially antigenic molecules into the systemic circulation of newborn animals by either facilitating the early maturation of intestinal barrier function or by providing passive barrier factors until the newborn's natural barrier can develop.
\end{abstract}

\section{Speculation}

The intestinal transport of macromolecules, including potential antigens, decreases with maturation of the intestinal mucosal barrier. Natural feeding, when compared with artificial feeding, may accelerate growth and maturation of intestinal barrier function, thereby decreasing the intestinal transport of antigens. This decreased transport of antigens with breast feeding may offer protection to the allergy-prone or otherwise disease-prone infant. In addition, reduced antigenic exposure during infancy may decrease the likelihood of sensitization which could be harmful later should gastrointestinal defense mechanisms be compromised by intestinal disease.
There is evidence that increased amounts of luminal antigens may cross the intestinal mucosal surface during the newborn period than at a later stage of life (13). This increased uptake of foreign macromolecules is most likely due to a delay in development of intestinal host defenses, both immunologic and nonimmunologic, collectively comprising the intestinal "mucosal barrier." In the absence of such mucosal barrier factors as adequate intraluminal proteolysis, secretory antibodies, or a mature intestinal epithelium to control macromolecular absorption, larger quantities of intact antigens penetrate the epithelium and pass into the lamina propria and intestinal circulation. However, enhanced intestinal permeability to macromolecules in neonates may also have a positive feature because in some species, it permits intestinal absorption of antibodies (1).

Colostrum has been reported to stimulate epithelial maturation in several organs including the intestine $(3,5)$ and is known to contain immunoglobulins directed against foreign antigens found in the maternal intestine (6). We have therefore hypothesized that ingestion of colostrum by newborns may facilitate mucosal barrier function and thereby interfere with the uptake of intestinal antigens.

The present study was undertaken to determine if newborn rabbits ingesting colostrum and breast milk from lactating does had decreased intestinal uptake of an antigen during the perinatal period compared to artificially fed animals. In addition, we conducted morphologic studies to determine if differences existed between the structure of newborn enterocytes in naturally fed rabbits compared to those fed an isocaloric formula to explain any transport changes noted.

\section{MATERIALS AND METHODS}

\section{ANIMALS}

Adult New Zealand White does raised on Charles River Rabbit Chow (Country Foods, Syracuse. NY), a plant-based diet devoid of BSA were mated. Naturally raised newborn animals (breast fed) were allowed to remain with the mother and suckle for 1 to 2 wk.

Artificially reared animals (bottle fed) were obtained by inducing labor in the pregnant doe on the 31st day of a 31-day term gestation by the intramuscular injection of 1.0 U.S.P. unit of oxytocin (Sandoz Pharmaceuticals, East Hanover, NJ) diluted to $0.2 \mathrm{ml}$ with water. The infant rabbits were removed from the doe at birth before suckling could take place, weighed, and placed in an incubator kept at 30 to $32^{\circ} \mathrm{C}$. Rabbits which weighed less than $40 \mathrm{~g}$ were considered low birth weight animals and were not used. The artificially reared animals were fed Esbilac, a commercially available fo:mula for mammals (Borden, Inc., Norfolk, VA). 
Table 1. Comparison of Esbilac' and rabbit milk

\begin{tabular}{|c|c|c|}
\hline Composition & $\begin{array}{l}\text { Esbilac powder } \\
\text { (diluted } 1: 1.5 \text { ) }\end{array}$ & Rabbit milk \\
\hline Crude protein & $13.0 \%$ & $11.0 \%$ \\
\hline Crude fat & $16.0 \%$ & $17.0 \%$ \\
\hline Carbohydrate & $6.0 \%$ & $2.0 \%$ \\
\hline Caloric density & $2.20 \mathrm{kcal} / \mathrm{ml}$ & $2.05 \mathrm{kcal} / \mathrm{ml}$ \\
\hline
\end{tabular}

'Borden Chemical, Borden, Inc., Norfolk, VA.

When the powdered Esbilac was diluted 1:1.5 with tap water, the diluted formula had a nutrient concentration approximating rabbit milk (Ref. 2: Table 1). A small nipple fitted into a hole constructed at the bottom of a disposable $12.0 \mathrm{ml}$ syringe was used to feed the newborn animals. Animals were fed every 4 to $8 \mathrm{hr}$.

Weight differences between one-wk-old naturally fed (101.0 \pm $7.0 \mathrm{~g} ; \overline{\mathrm{x}} \pm \mathrm{S}$.E. $)(N=15)$ and artificially raised animals $(106.0 \pm$ $4.0 \mathrm{~g})(N=12)$ were not significant. By $2 \mathrm{wk}$ of age, the body weights of both groups [natural $=230.0 \pm 16.0 \mathrm{~g}(N=6)$ and artificial $=212.0 \pm 9.0 \mathrm{~g}(N=6)$, respectively] were also quite similar $(P$, n.s. $)$.

\section{TRANSPORT STUDIES}

Animals were fasted overnight and then gavaged with $100 \mathrm{mg}$ of crystalline BSA (Sigma Biochemical Co.. St. Louis, MO) mixed in one $\mathrm{ml}$ of phosphate-buffered saline. Because it had previously been established that the maximum concentration of plasma IBSA after BSA gavage occurred at $4 \mathrm{hr}$ (13), blood was obtained by cardiac puncture $4 \mathrm{hr}$ after tube feeding. Serum immunoreactive BSA concentrations were determined on aliquots of samples using an electroimmunodiffusion technique (E.I.D.) (8) as previously described (13).

To evaluate the possibility that naturally raised does might have been exposed to BSA resulting in breast milk antibodies to this protein, which could possibly interfere with BSA transport. counterimmunoelectrophoresis (7) was performed to determine if antiBSA activity was present in breast milk. Likewise, plasma of selected naturally and artificially raised animals was examined with counterimmunoelectrophoresis and hemagglutination (4) to determine if anti-BSA activity was present. In addition. both types of milks and plasma from selected breast- and bottle-fed animals not used in the BSA transport study were tested for the presence of I-BSA using E.I.D. and/or counterimmunoelectrophoresis.

Statistics: All determination of statistical significance were made using the Student $t$ test.

\section{MORPHOLOGIC STUDIES}

One and 2-wk-old breast- and bottle-fed rabbits were anesthetized with ether, and segments of proximal and distal small intestine were obtained. All intestinal tissue was dissected free from mesentary, cut into one mm slices, and immersed for one to $2 \mathrm{hr}$ either in $2.5 \%$ cacodylate-buffered glutaraldehyde with $1.5 \%$ paraformaldehyde and $0.01 \mathrm{M} \mathrm{CaCl}_{2}$ or in $1 \%$ dichromatebuffered osmium tetroxide $\left(\mathrm{OsO}_{4}\right)$, both at $\mathrm{pH}$ 7.4. Tissue fixed in glutaraldehyde was rinsed with cacodylate buffer for $24 \mathrm{hr}$ and postfixed for one hr in 1\% cacodylate-buffered $\mathrm{OsO}_{4}$. Tissue fixed in $\mathrm{OsO}_{4}$ was postfixed for $30 \mathrm{~min}$ in formalin. Tissue was then dehydrated and embedded in epon 812 (9). Sections $1.0 \mu \mathrm{m}$ thick were cut with a Porter Blum MT2B microtome, mounted on glass slides, and stained with methylene blue azure II (12). Tissue blocks with well-oriented villi were trimmed, thin sectioned on an LKB ultramicrotome, contrasted with uranyl acetate and lead citrate. and examined with a Philips 300 electron microscope.

\section{RESULTS}

\section{TRANSPORT STUDIES}

A significant difference was noted in plasma I-BSA $4 \mathrm{hr}$ after BSA gavage in 1-wk-old breast- and bottle-fed animals (Fig. 1).

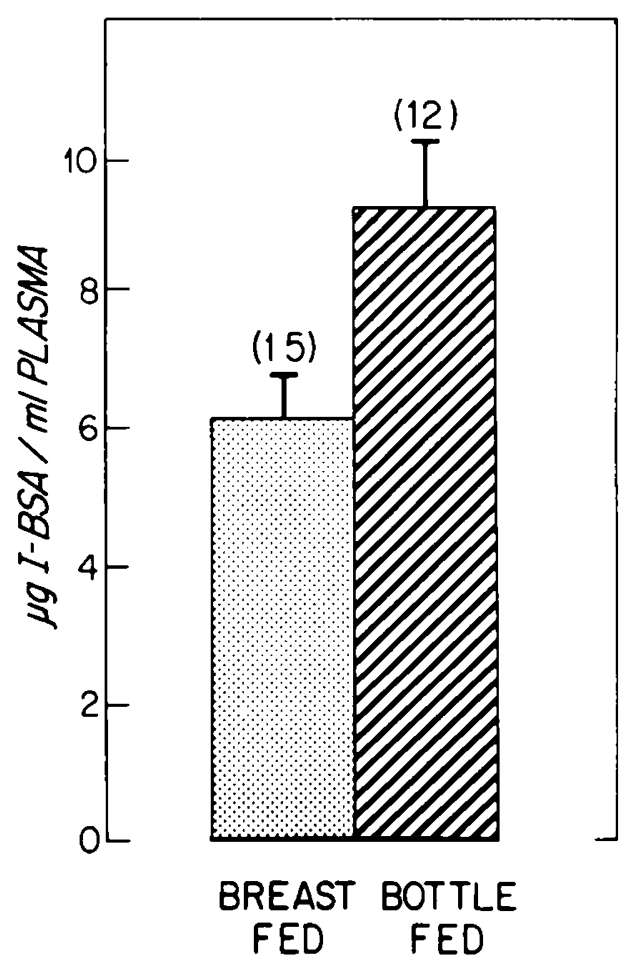

Fig. 1. Immunoreactive $\mathrm{BSA}(\mathrm{ugm} / \mathrm{ml})$ in plasma obtained from 1 -wkold breast- $(N=15)$ and bottle-fed $(N=12)$ rabbits $4 \mathrm{hr}$ after gavage feeding $100 \mathrm{mg} \mathrm{BSA}$.

One-wk-old breast-fed animals had $6.12 \pm 0.77 \mu \mathrm{g}$ I-BSA per ml plasma, and bottle-fed animals had $9.19 \pm 0.93 \mu \mathrm{g}$ l-BSA per ml $(P<0.02)$. None of the bottle-fed animals $(N=6)$ at $2 \mathrm{wk}$ of age had detectable plasma I-BSA $4 \mathrm{hr}$ after BSA gavage, and four of six breast-fed animals had barely detectable plasma l-BSA. (Mean I-BSA for the 2-wk-old breast-fed animals was $<1.0 \mu \mathrm{g} \mathrm{I-BSA} \mathrm{per}$ $\mathrm{ml}$ ).

Using counterimmunoelectrophoresis, no anti-BSA could be detected in breast milk of lactating does. Likewise, no anti-BSA could be detected by counterimmunoelectrophoresis or hemagglutination in the plasma of one and 2-wk-old breast- and bottle-fed animals. In addition, no I-BSA was identified using E.I.D. and/or counterimmunoelectrophoresis in either breast or formula milk or in the plasma of naturally and artificially fed animals not gavage fed BSA.

\section{MORPHOLOGIC STUDIES}

In l-wk-old rabbits, there was no obvious structural differences between bottle- and breast-fed pups (Figure 2, $A$ and $B$, respectively). In absorptive cells of proximal intestine, the apical tubular system and lysosome-like structures were present but varied substantially in extent from cell to cell. Coated pits were occasionally noted along the apical, lateral, and basal plasma membranes, and coated vesicles were seen throughout the cytoplasm. There were putative lipid droplets in the smooth endoplasmic reticulum, and chylomicrons were abundant in the golgi complex, the lateral intercellular spaces, and the lamina propria. Unusual arrays of membranes of unknown significance but perhaps related to smooth endoplasmic reticulum were prominent in the apical cytoplasm of many cells of both breast- and bottle-fed animals (Fig. $2 A$ ). These appeared as closely opposed tubules arranged in an interwoven, crystalloid pattern or wavy swirl-like aggregates. Pseudopod-like cytoplasmic extensions, similar to those in developing rat intestine $(10)$, penetrated the basal lamina and projected into the lamina propria from the basal surface of many absorptive cells (Fig. 3).

Compared to the proximal intestine, the absorptive cells of distal intestine in both breast- and bottle-fed animals at 1 wk were characterized by a more extensive apical tubular system and 


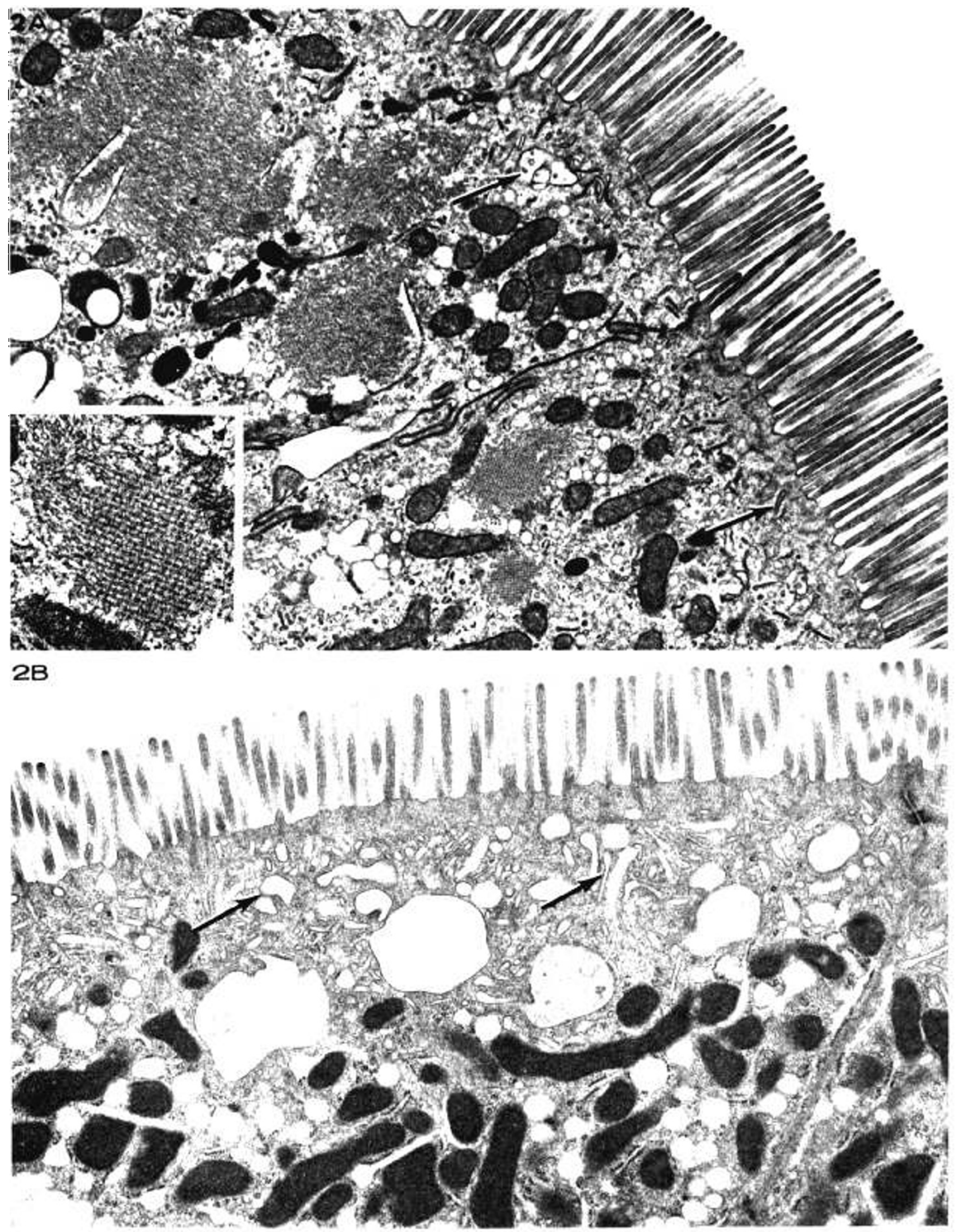

Fig. 2. A, apical cytoplasm from the proximal small intestine of a 1-wk bottle-fed rabbit. Numerous vesicles and tubules (arrows) are present beneath the microvillous border. The remainder of the cytoplasm is characterized by small electron dense lysosomal elements, mitochondria, small lipid droplets, and unusual arrays of membranes. $\times 13.000$.

Inset, higher magnification of one of the membranous arrays seen in $A$. Closely opposed tubules are continous with an interwoven crystalloid pattern of membranes. $\times 30,200$.

$B$, apical cytoplasm from the proximal small intestine of a I-wk breast-fed rabbit. Abundant profiles of tubules, vesicles, and vacuoles (arrons) are present along with numerous mitochondria and lipid droplets. The membranous arrays are absent from this particular section but were seen in others. $(\times 14.900)$. 


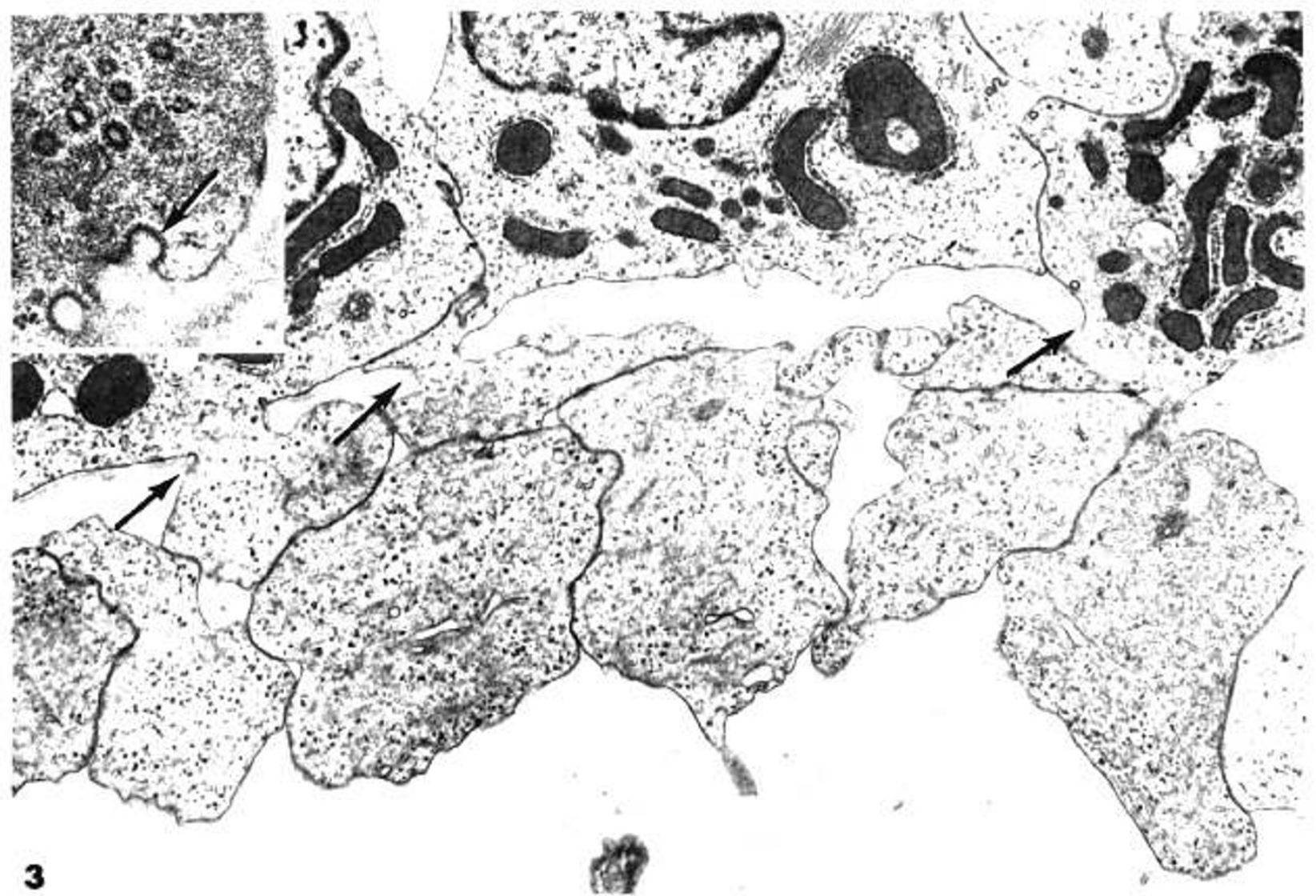

Fig. 3. Basal cytoplasm of several absorptive cells from the proximal small intestine of a one-wk breast-fed rabbit. Pseudopod-like extensions of cytoplasm penetrating through the basal lamina (arrows) and extending into the lamina propria. Many of these pseudopodia are closely opposed to similar projections from the same and adjacent cells. $\times 8,300$

Inset, higher magnification of the basal cytoplasm of an absorptive cell from an area with many pseudopodia. There are several coated vesicles in the cytoplasm and a coated pit at the base of the cell (arrow). $\times 37,100$.

substantially larger lysosomal elements. Some differences were noted in the staining characteristics of the lysosomal elements in the breast- versus bottle-fed neonates, perhaps a reflection of dietary differences. As in the proximal intestine, chylomicrons were observed in the intercellular spaces and lamina propria. In contrast, the unusual membranous arrays seen in proximal intestine were not present and pseudopodia were extremely rare.

At 2 wk of age, the morphologic appearance of the proximal and distal intestine did not differ appreciably from the 1 wk samples.

\section{DISCUSSION}

This study provides direct evidence that breast-fed pups are protected from enhanced antigen penetration across the intestinal mucosal barrier in the immediate postpartum period compared to artificially fed animals. Because a significant decrease in circulating BSA was noted in 1-wk-old naturally fed animals compared to artifically fed controls, this difference in BSA transport may be attributable to protection provided by colostral ingestion. By $2 \mathrm{wk}$ postpartum, however, uptake of BSA in both groups of animals decreased to the limit of detection ( $1.0 \mu \mathrm{g}$ I-BSA per $\mathrm{ml}$ plasma), suggesting that maturation of the mucosal barrier results in control of antigen uptake from the intestinal lumen.

Dilution of transported I-BSA in the vascular compartment, may in part account for the observed decrease in plasma levels between 1 and 2 wk in both groups. However, a 50\% decrease in plasma I-BSA concentration would be predicted from 1 to $2 \mathrm{wk}$ of age if dilution were the only contributing factor because vascular volume doubles during that time (11). A 50\% decrease in our reported 1 -wk-old levels would give plasma levels of 3 and 4.5 $\mu \mathrm{g} / \mathrm{ml}$, respectively, in the breast- and bottle-fed groups at $2 \mathrm{wk}$ of age. This level is substantially greater than the actual mean values obtained $(<1.0 \mu \mathrm{g} \mathrm{I-BSA}$ per $\mathrm{ml})$ for the respective groups and suggests factors other than dilution account for the decreased plasma levels in the 2-wk-old group.

The possibility that I-BSA was cleared from the vascular space more rapidly in breast-fed animals seems unlikely because in studies not reported here we have noted that the same amount of intravascularly injected I-BSA remained in both the natural- and artificially fed animals after $4 \mathrm{hr}$.

Decreased intestinal transport of I-BSA at $1 \mathrm{wk}$ in the naturally fed animals does not seem to be mediated by immune exclusion mechanisms because no anti-BSA antibodies could be demonstrated by counterimmunoelectrophoresis in the breast milk of lactating does. Although the possibility of secretory antibodies against BSA present on mucosal surfaces cannot be ruled out, this possibility seems unlikely because no BSA could be identified in either breast milk or artificial formula by counterimmunoelectrophoresis. However, it is conceivable that concentrations of antiBSA antibodies may be present in breast milk and plasma at levels undetected by counterimmunoelectrophoresis and hemagglutination. In addition, cell-mediated immunologic mechanisms acting to decrease transport of antigen in the breast-fed animals cannot be eliminated because this was not evaluated.

Although not studied, nonimmunologic factors known to be important in barrier function should be considered as an explanation for the decreased antigen transport in 1-wk-old breast-fed animals. Possibilities include the accelerated maturation of releasing mechanisms for pancreatic enzymes and/or intraluminal enzymes important to protein hydrolysis, changes in gastric and/or intestinal $\mathrm{pH}$, changes in the glycocalyx or mucous secretion in bottle-fed animals more conductive to antigen transport, and finally, the induction of lysosomal enzymes in breast-fed animals 
which contributes to the intracellular breakdown of absorbed proteins and thereby decreases the intact protein delivered to the systemic circulation of these animals. Further studies are needed to evaluate these possibilities.

No striking difference in morphologic characteristics between naturally and artificially fed animals could be noted. However, careful quantitative morphometric studies would be needed to exclude subtle changes between the two groups. Furthermore, substantial maturational changes might occur in the absence of obvious morphologic changes, as for example, changes in the composition of the glycocalyx. Therefore, the absence of morphologic changes per se does not entirely exclude the possibility of maturational events accounting for transport differences.

In summary, this study suggests that factors present in breast milk may be operating to enhance intestinal mucosal barrier function in the breast-fed newborn, thereby decreasing early systemic exposure to antigens.

\section{REFERENCES AND NOTES}

I. Abrahamson. D. R.. Powers, A., and Rodewald. R.: Intestinal absorption of immune complexes by neonatal rats: A route of antigen transfer from mother to young. Science (Wash. D. C.). 206: 567 (1979).

2. Cowie. A. T.: Variations in the yield and composition of the milk during lactation in the rabbit and the galactopoietic effect of prolactin. J. Endocrinol.. 44: 437 (1969).

3. Hall. R. A.. And Widdowson. E. M.: Response of the organs of rabbits to feeding during the first days after birth. Biol. Neonate. 35: 131 (1979).

4. Herbst. W. J.: Passive hemagglutination with special reference to the tanned cell

Copyright $(1981$ International Pediatric Research Foundation, Inc. $0031-3998 / 81 / 1503-0245 \$ 02.00 / 0$ technique. In: D. M. Weir: Handbook of Experimental Immunology. Vol. I. p. 20. 1 (Blackwell Scientific Publications. Oxford. 1978).

5. Klagsbrun, M.: Bovine colostrum supports the serum free proliferation of epithelial cells but not of fibroblasts in long term culture. J. Cell Biol.. 84: 808 (1980).

6. Kleinman, R. E., and Walker, W. A.: The enteromammary immune system: An important new concept in breast milk host defense. Dig. Dis. Sci.. 24: 876 (1979).

7. Kohn. J.: Immunoelectroosmophoresis. Methods Immunol. Immunochem. 3: 277 (1971).

8. Laurell. C. B.: Quantitative estimation of proteins by electrophoresis in agarose gel containing antibodies. Anal. Biochem., 15: 45 (1966).

9. Luft. J. H.: Improvements in epoxy resin embedding methods. J. Biophys. Biochem. Cytol., 9: 409 (1961).

10. Mathan. M.. Hermos. J. A., and Trier. J. S.: Structural features of the epitheliomesenchymal interface of rat duodenal mucosa during development. J. Cell Biol.. 52: 577 (1972).

11. Mott. J. C : Haemorrhage as a test of the function of the cardiovascular system in rabbits of different ages. J. Physiol. (Lond.), 181: 728 (1965).

12. Richardson. K. C.. Jarretı. L. K.. and Fincke, E. H.: Embedding epoxy resins for uitrathin sectioning in electron microscopy. Stain Technol.. 35: 313 (1960).

13. Udall, J. N., Fritze. L., Pang, K.. Kleinman, R., and Walker. W. A.: Development of gastrointestinal mucosal barrier. 1 . The effect of age on intestinal permeability to macromolecules. Pediatr. Res.. 15: 214 (1981).

14. Dr. Udall was the recipient of a National Institutes of Health Research Training Fellowship in Clinical Nutrition (T-32-AM-07070).

15. This study was presented in part at the annual meeting of the Society for Pediatric Research, May, 1979. Atlanta, Georgia.

16. Requests for reprints should be addressed to: J. N. Udall. M.D.. Pediatric Gastrointestinal and Nutrition Unit. Children`s Service. Massachusetts General Hospital. Boston. Massachusetts 02114.

17. This research was supported in part by grants (HD-12437. AMDD-16269. (iM21700 , and AMDD-17537) from the National Institutes of Health.

18. Received for publication April 30, 1980

19. Accepted for publication August 4, 1980 